\title{
TENSILE AND IMPACT BEHAVIOR OF REINFORCED COMPOSITE MATERIAL BY HAND LAY-UP TECHNIQUE
}

\author{
Pothamsetty Kasi V Rao ${ }^{1}$, B. Akhil Babu ${ }^{2}$, A. L. V. N. Gupta ${ }^{3}$ \\ ${ }^{1}$ Assistant Professor, Mechanical Engineering, K L University, Guntur, AP, India \\ ${ }^{2}$ Student, Mechanical Engineering, K L University, Guntur, AP, India \\ ${ }^{3}$ Student, Mechanical Engineering, K L University, Guntur, AP, India
}

\begin{abstract}
Our work mainly focuses on converting waste materials into raw materials and to increase the tensile and impact behavior of fiber reinforced polymer composite. The study has been carried out in view of high lightening advantages of natural fibers over man-made or synthetic fibers. In this work polyester is used as a matrix and corchorous plant fiber i.e. Jute is used as a reinforcing material. Tensile test specimen is made as per ASTM-D638M-89 and Impact test specimen is made as per ASTMD256M-97. Material properties for both tensile strength and impact strength are obtained by considering different weights of reinforced material with matrix phase. In this paper the methodology of preparation of mould and composite using hand lay-up technique was presented.
\end{abstract}

Keywords: Natural Fiber, Synthetic Fiber, Polyester, Corchorous Plant Fiber, Tensile Test Specimen, Impact Test Specimen.

\section{INTRODUCTION}

In the proceeding with journey for enhanced execution, which may be determined by different criteria including less weight, more quality and lower expense, as of now utilized materials oftentimes achieve the point of confinement of their convenience. Along these lines material researchers, designers and researchers are continually endeavoring to create either enhanced customary materials or totally new materials. Composites are an illustration of the last class. Inside of last forty to fifty years, there has been a fast increment in the creation of engineered composites, those fusing fine strands in different plastics (polymers) commanding the business sector. With the expanding worldwide vitality emergency and biological dangers, researchers everywhere throughout the world are moving their consideration towards elective answer for engineered fiber.

Since 1990s, common fiber composites are rising as sensible distinct option for glass-strengthened composites in numerous applications. Characteristic fiber composites are asserted to offer natural favorable circumstances, for example, decreased reliance on non-renewable vitality/material sources, lower contamination discharges, lower green house gas outflows, upgraded vitality recuperation and end of life biodegradability of parts. Such unrivaled ecological exhibitions are imperative driver of expanded future utilization of normal fiber composite.

India invested with a copious accessibility of common fiber, for example, Jute, Coir, Sisal, Pineapple, Ramie, Bamboo, Banana and so on has concentrated on the improvement of regular fiber composites essentially to investigate quality included application parkways. Such characteristic fiber composites are appropriate as wood substitutes in the lodging and development part. The advancement of normal fiber composites in India depends on two dimensional systems of averting consumption of timberland assets and in addition guaranteeing great monetary returns for the development of regular strands.

The advancements in composite material in the wake of meeting the difficulties of aviation area have fell down for taking into account local and modern applications. Composites, the miracle material with light-weight; high quality to-weight proportion and firmness properties have progress significantly in supplanting the traditional materials like metals, wood and so forth. The material researchers everywhere throughout the world centered their consideration on characteristic composites strengthened with Jute, Sisal, Coir, and Pineapple and so on essentially to chop down the expense of crude materials.

\subsection{Definition of Composite}

A composite is blend of two or more diverse materials that result in better properties than those of individual composites used alone. IN contrast of metallic alloys, each material retains its separate physical, chemical and mechanical properties. The main advantage of composite materials are its high strength and stiffness combined with low density, when compared with bulk materails.

The Reinforcing phase or fiber phase which is discontinuous in composites is stronger and stiffer than the continuous matrix phase. The lattice stage goes about as a heap exchange medium between one fiber and another fiber, and 
sometimes where the heaps or weights are perplexing; the grid may even need to hold up under burdens transverse to the fiber pivot. The framework stage is more flexible than the fiber stage and hence goes about as a wellspring of sturdiness. The grid additionally acts to shield the filaments from natural harm amid and after composite preparing.

\subsection{Classification}

The commonly accepted composites are

- Fibrous Composites

- Laminated Composites

- Particulate Composites

The main types of fibers are

- Natural Fibers Ex: Jute, Coir, Baggase, Corn etc

- Man Made Fibers Ex: Carbon, Boron, Glass, Graphite

\section{LITERATURE REVIEW}

Sreenivasan et al took a shot at the advancement of thermoplastic elastomer composite fortified with $20 \mathrm{vol}$. \% kenaf fiber with different mixes like polypropylene thermoplastic natural rubber and polypropylene/ethylenepropylene-diene-monomer (PP/EPDM) that were delivered through twofold liquefy mixing strategy [1].

H. Anuar , A. Zuraida worked on mechanical and thermal properties of natural fibers such as sisal, hemp, coir, kenaf and jute reinforced polymer composites. The tensile properties have been studied, and reported that among all the composites, hemp reinforced composite exhibited the highest mechanical properties whereas the coir showed the lowest [2].

N. Venkateshwaran et al has tentatively discovered rigidity and modulus of short, haphazardly arranged cross breed normal fiber composite furthermore anticipated utilizing Rule of Hybrid Mixture (RoHM). Mixture composites were readied utilizing banana/sisal strands of 40:0, 30:10, 20:20, 10:30, and 0:40 proportions, while general fiber volume part was settled as $0.4 \mathrm{Vf}$ and the correlation in the middle of exploratory and RoHM demonstrated that they are in great assention [3].

T. Vijaya kumar et al in their work used polyester as a lattice and banyan tree fiber as a fortifying material. Elastic test example is made according to ASTM D638I. Material properties of the composite have been concentrated on with the assistance of diverse rate weight proportions of lattice to fiber. Additionally the quality of composite is evaluated with the variety of fiber length [4].

Nguyen Tri Phuong et al in their study found that Reinforced composites acquired with acetylated BF demonstrate better mechanical properties because of joining of acetyl gatherings onto the cellulose fiber surface and along these lines enhance similarity in the middle of BF and network. The rheological properties of RPP/BF composites relying upon the BF substance and treatment techniques are additionally examined. Prevalent variables that impact the properties of pertinent materials are distinguished. Maleic anhydride united polypropylene is utilized as a compatibilizer to enhance the grip between the cellulosic stage and the RPP grid [5].

S.Chapple in his work examined important aspects of the flammability of natural fiber reinforced composites and to outline some of the more recent strategies used to improve their performance [6].

V Ratna Prasad worked on mechanical properties of bananaEFB and being a low density fiber $(581 \mathrm{~kg} / \mathrm{m} 3)$ as compared to those of sisal, jute, coir and bamboo $(>1000$ $\mathrm{kg} / \mathrm{m} 3$ ), the composite can be regarded as a useful lightweight engineering material. Hence, without any additional cost input, the banana-EFB fiber/polyester composites can be used for industrial applications like partition panels, packaging and automotive industry [7].

Flavio de Andrade Silva have studied the monotonic tensile behavior of a high performance sisal fiber and Nitinat Suppakarn used magnesium hydroxide $\left(\mathrm{Mg}(\mathrm{OH})_{2}\right)$ and zinc borate, as flame retardants, were incorporated into sisal/PP composites. Maleic anhydride grafted polypropylene was also used as a compatibilizer. Good distribution of flame retardants and sisal fiber in PP matrix was also observed [8].

\section{MATERIALS AND METHODS}

The general purpose polyester resin of ECMALON 4413 grade was procured from ECMAS RESIN private limited, Hyderabad. It is an unsaturated resin having viscosity of 500-600CPS (Brookfield Viscometer) and specific gravity of $1.13 \mathrm{gm} / \mathrm{cc}$ at $25^{\circ} \mathrm{C}$.

\subsection{Extraction and Preparation of Jute Fiber}

Jute is a long soft shiny vegetable fiber, produced from the Co chorus plant. The fiber is spun into coarse, strong threads and used for a variety of commercial purposes. . The fiber is an off white to brown color, and range from to 3-12 feet long. The course of each fiber is made into small short length fibers i.e. $40 \mathrm{~mm}, 50 \mathrm{~mm}$ etc.

\subsection{Hand lay-up Technique}

Hand lay-up is the least difficult and most established technique for the composite manufacture forms. It is a low volume, work escalated strategy suited particularly for vast segments, for example, vessel frames. The contact shaping strategy comprises of applying these components progressively onto a mould surface, are lease specialists, a gel coat, a layer of fluid thermosetting resin, of viscosity around 0.3 and $0.4 \mathrm{~Pa} . \mathrm{s}$, and of medium reactivity, a layer of support (glass, carbon, and so on.) as slashed strand mat or woven roving impregnation of the fortification is finished by hand utilizing a roller or a brush. This operation is rehashed for every layer of fortification keeping in mind the end goal to get the fancied thickness of the structure. 


\section{Hand Layup}

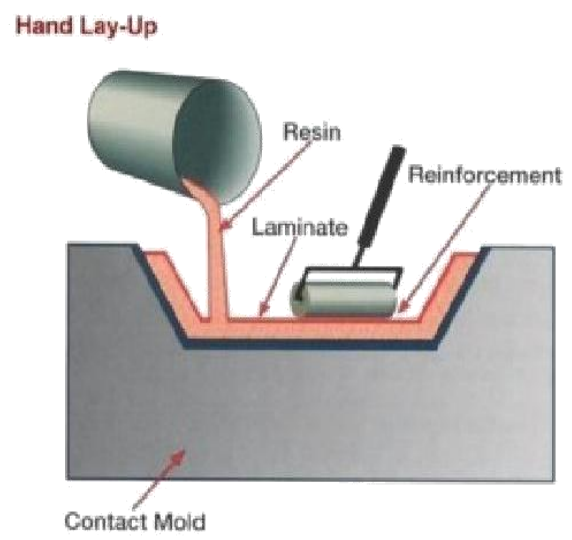

Fig 1: Hand Lay-up Technique

\subsection{Fabrication and Testing of Composites}

Different steps are involved in making of composites such as collection of fibers and resins, preparation of mould as per standard dimensions i.e. ASTM-D638M-89, making and extraction of composite from the mould.

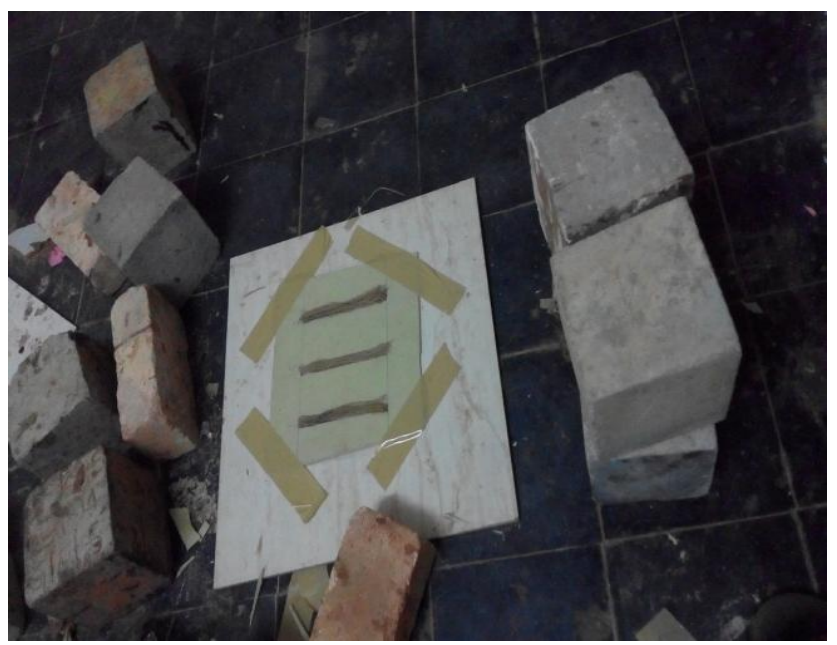

Fig 2: Fabrication of Composite Materials

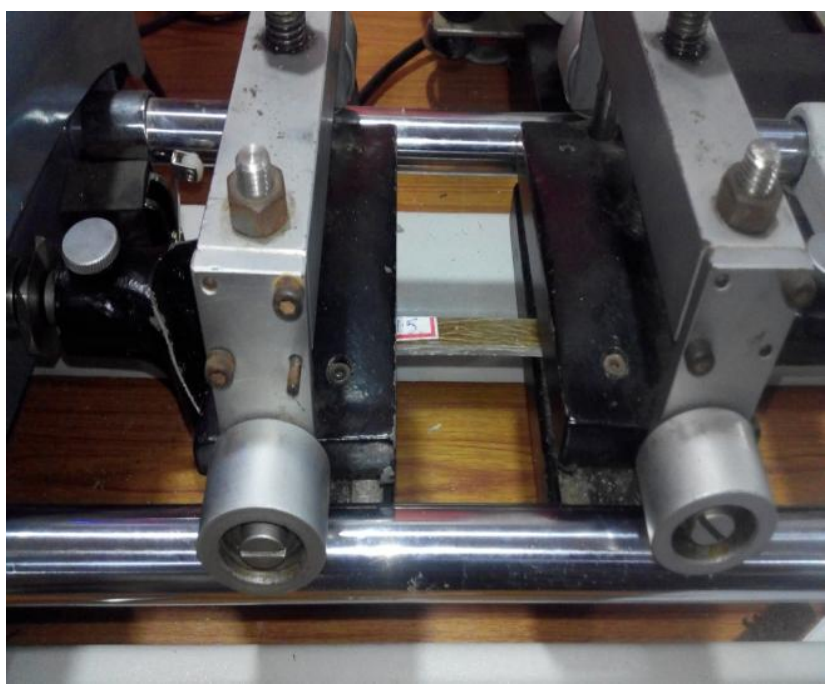

Fig 3: Composite under Tensile Strength

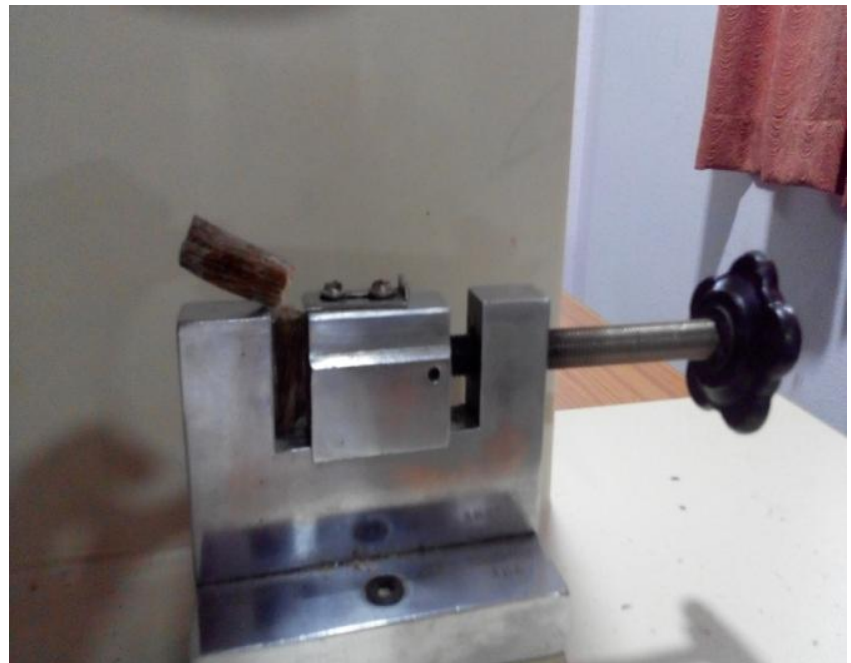

Fig 4: Composite under Impact Strength

\section{RESULTS AND DISCUSSION}

\subsection{Tensile Testing}

The below table shows the values of tensile strength obtained for the dimensions as per ASTM-D638M-89 standard of tensile testing for different weights of jute fiber( $0 \mathrm{gm}, 1 \mathrm{gm}, 2 \mathrm{gm})$ with mixing of polyester reinforced composite.

Table 1: Tensile Strength for different weights of Jute Fiber

\begin{tabular}{|c|c|c|}
\hline $\begin{array}{c}\text { ACTUAL } \\
\text { WEIGHT } \\
\text { FIBER }(\mathrm{gm})\end{array}$ & $\begin{array}{c}\text { MEAN } \\
\text { WEIGHT } \\
\text { FRACTION }\end{array}$ & $\left.\begin{array}{c}\text { MEAN TENSILE } \\
\text { STRENGTH(N/mm }\end{array}{ }^{2}\right)$ \\
\hline 2 & 17.30 & 47.17 \\
\hline 1 & 9.925 & 30.16 \\
\hline 0 & 0 & 17.00 \\
\hline
\end{tabular}

The below graph shows the graph between Mean weight fraction taken on $\mathrm{X}$-axis and Mean tensile strength taken on Y-axis

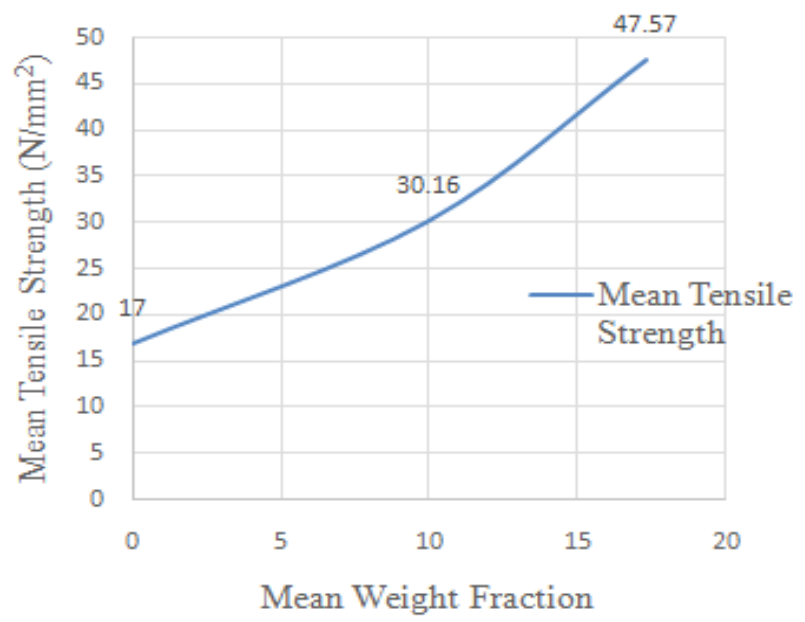

Fig 5: Graph for tensile strength vs Mean weight fraction 


\subsection{Impact Testing}

The below table shows the values of impact strength obtained for the dimensions as per ASTM-D256M97standard of impact testing for different weights of jute fiber(0gm, 1gm, $2 \mathrm{gm})$ with mixing of polyester reinforced composite.

Table 1: Impact Strength for different weights of Jute Fiber

\begin{tabular}{|c|c|c|}
\hline $\begin{array}{c}\text { ACTUAL } \\
\text { WEIGHT } \\
\text { FIBER(gm) }\end{array}$ & $\begin{array}{c}\text { MEAN } \\
\text { WEIGHT } \\
\text { FRACTION }\end{array}$ & $\begin{array}{c}\text { MEAN IMPACT } \\
\left.\text { STRENGTH(N/mm }{ }^{2}\right)\end{array}$ \\
\hline 2 & 25.19 & 260 \\
\hline 1 & 18.23 & 196.6 \\
\hline 0 & 0 & 30 \\
\hline
\end{tabular}

The below graph shows the graph between Mean weight fraction taken on $\mathrm{X}$-axis and Mean impact strength taken on Y-axis

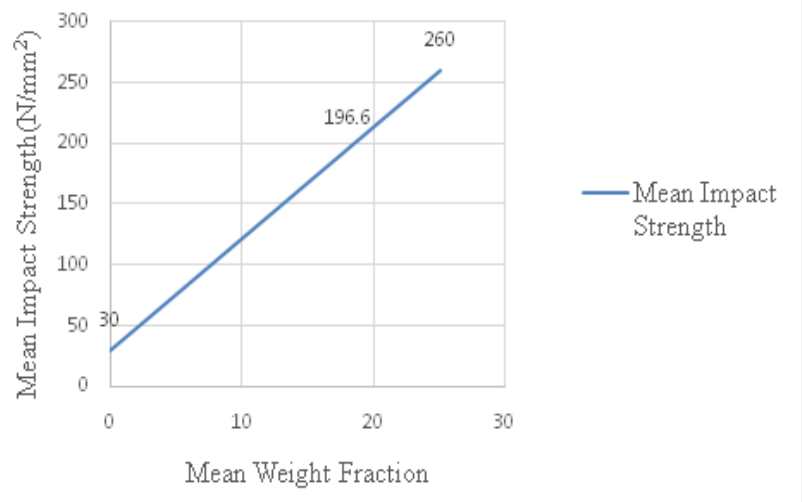

Fig 6: Graph for Impact strength vs Mean weight fraction

\section{CONCLUSION}

The Tensile Strength and Impact Strength values for Jute fiber reinforced polymer composite were found to be increased with the increase in weight fraction of Jute fiber. Hence it is understood that the composite materials strength lies in the selection of proper reinforcing material with good fabrication techniques. The work can be further extended to determine other mechanical properties for different weight fractions of jute fiber.

\section{REFERENCES}

[1] Sreenivasan, Soma Sundram S, Ravindran D, Manikandan V, Naryanaswamy R, "Micro structural, physic-chemical and mechanical characterization of sansevieria cylindrical fibers-An exploratory investigation" Materials and design, vol. 32 , 2011, pg: 453.
[2] H. Anuar , A. Zuraida, "Improvement in mechanical properties of reinforced thermoplastic elastomer composite with Kenaf bast fiber", composites, vol.42, 2011,pg: 462-465.

[3] N. Venkateshwaran , A. Elayaperumal, G.K. Sathiya, “ Prediction of tensile properties of hybrid-natural fiber composites", composites: part b, vol.43, 2012,pg: 793 796.

[4] T. Vijaya kumar, Dr. K. V. Ramana, Dr. R B Chowdary, "Tensile behavior of banyan tree fiber reinforced composites" International Journal of Advanced Engineering Research and Studies vol.1(2), 2012, pg: 256-258.

[5] Nguyen Tri Phuong, Cyrille Sollogoub and Alain Guinault, "Relationship between fiber chemical treatment and properties of recycled pp/bamboo fiber composites", Journal of reinforced plastics and composites vol.29(21), 2010, pg: 3244-3256.

[6] S.Chapple, and R. Anandjiwala, "Flammability of natural fiber-reinforced composites and strategies for fire retardancy", journal of thermoplastic composite materials, vol.23, 2010, pg: 871.

[7] V Ratna Prasad, K Mohana Rao \& G Nagasrinivasulu," Mechanical properties of banana empty fruit bunch fiber reinforced polyester composites", Indian journal of fiber \& textile research, vol. 34, 2009, pg: 162-167.

[8] Flavio De Andrade Silva A, Nikhilesh Chawla B , Romildo Dias De Toledo Filho A," Tensile behavior of high performance natural (sisal) fibers", composites science and technology, vol.68, 2008, pg: 3438-3443. 